\title{
Laparoscopic extravesical neoureterocystostomy and vesicopsoas hitch for infiltrative ureteric and vesical endometriosis: case report
}

Received: 16 April 2006 / Accepted: 8 June 2006 / Published online: 2 August 2006

(C) Springer-Verlag Berlin / Heidelberg 2006

\begin{abstract}
We present a multidisciplinary approach to the management of a 41-year-old woman who presented with an acute on chronic history of pelvic pain and urinary tract symptoms. The underlying pathology was found to be infiltrative ureteric and vesical endometriosis. The extent of the disease had caused partial ureteric obstruction. The patient subsequently underwent laparoscopic excision of the endometriosis with a laparoscopic extravesical neoureterocystostomy and vesicopsoas hitch, performed by an advanced laparoscopic gynaecologist and a urologist.
\end{abstract}

Keywords Laparoscopy · Ureteric · Vesical · Endometriosis $\cdot$ Neoureterocystostomy $\cdot$ Vesicopsoas hitch

\section{Case report}

A 41-year-old multigravid women presented with a 3month history of right loin pain, dysuria and haematuria. A computed tomography (CT) scan reported a $2.4-\mathrm{cm}$ thickening of the bladder and the patient subsequently underwent cystoscopy and ureteroscopy in May 2005. This showed a 2- to $3-\mathrm{cm}$ endometriotic nodule involving the bladder mucosa near the right ureteric orifice (Fig. 1) with a $3-\mathrm{cm}$ narrowing of the right ureter. A double-J stent was passed through the right ureter and a laparoscopic excision of this nodule with ureteric reimplantation planned.

In May 2001, a laparoscopic vaginal assisted hysterectomy (LAVH) was performed for dysmenorrhoea and menorrhagia. Histology of her uterus showed adenomyo-

K. Johnston $(\bowtie) \cdot$ A. Shukla $\cdot$ B. Jones $\cdot$ S. Sowter $\cdot$ D. Chou Sydney Women's Endosurgery Centre,

St George Private Hospital, Ground Level,

2217 Sydney, Kogarah, New South Wales, Australia

e-mail: keithmjohnston@bigpond.com

P. Aslan

Urology Sydney, St George Private Hospital, Level 1,

2217 Sydney, Kogarah, New South Wales, Australia sis. Due to persistent pain, a laparoscopy was performed 1 year later and a nodular deposit of endometriosis was seen on the right fundal aspect of the bladder near the ureteric tunnel. A gonadotrophin-releasing hormone analogue was tried but failed to resolve the pain. Cystoscopy performed at the same time demonstrated no endometriotic bladder mucosal involvement. In May 2003 the patient underwent a laparoscopic excision of a 3- to $4-\mathrm{cm}$ endometriotic bladder nodule and opted to have a bilateral salpingo-oophorectomy at the same time. Cystoscopy up to this stage was normal.

A four-port laparoscopy was performed with cystoscopy to help define the margins of the lesion from an abdominal and vesical perspective. Retroperitoneal dissection of the ureter from the bladder to about $6 \mathrm{~cm}$ above the pelvic brim was performed and the endometriotic ureter transected about $3 \mathrm{~cm}$ distal to its vesical insertion, exposing the double-J stent (Figs. 2 and 3). The proximal end of the ureter was examined and found to be free of endometriosis with a good vascular supply. The vagina was dissected free of the bladder to allow for precise resection of the endometriotic nodule. This was then excised using high power density monopolar diathermy. Despite ureteric dissection there was felt to be too much tension to reanastomose the ureter to the bladder and a laparoscopic vesicopsoas hitch was then performed as described below.

The anterior abdominal wall peritoneum was retracted vertically down at the level of the median umbilical ligament and opened to access the cave of Retzius. Dissection of the cave of Retzius then allowed adequate mobilization of the bladder toward the right psoas muscle, which had been exposed with careful dissection. Two sutures (1-0 Polyglycol Vicryl) were then used to secure the bladder dome to the tendon of the psoas muscle (Fig. 4). The defect in the bladder was closed using interrupted 3-0 polydioxanone sutures leaving a small neocystomy (Fig. 5). The proximal end of the ureter was spatulated and a full thickness tension-free anastomosis to the neocystomy with 4 interrupted 3-0 polydioxanone sutures at 3, 6, 9 and 12 o'clock positions (Fig. 6). The double-J stent was left in situ. Indigo carmine was given 


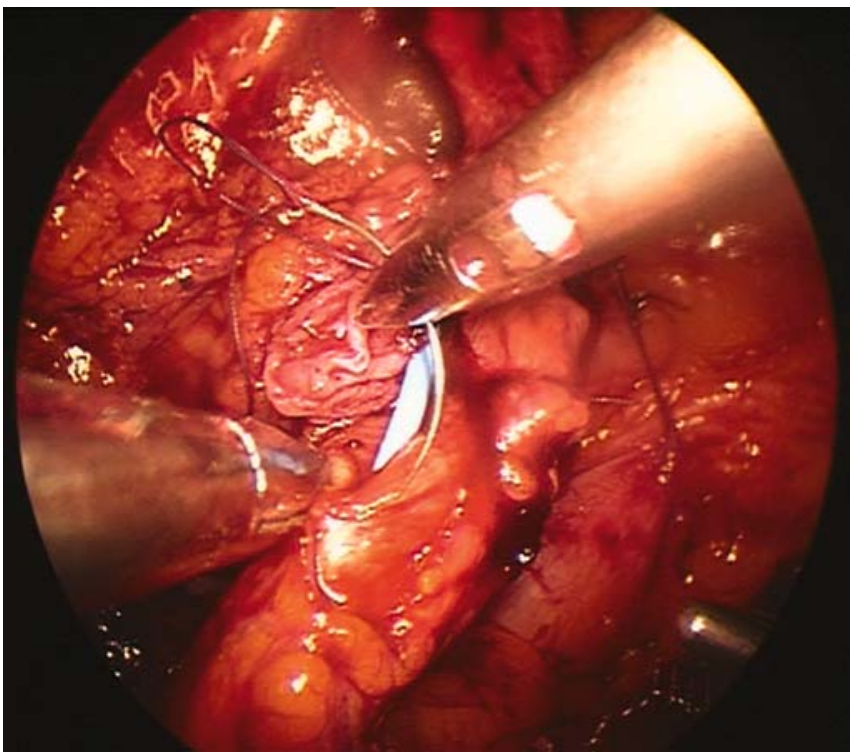

Fig. 1 Endometric bladder nodule

intravenously and this confirmed bilateral ureteral patency. A drain was left in the pouch of Douglas for $48 \mathrm{~h}$. An intracorporeal suturing technique was used throughout the operation.

The operation time was $210 \mathrm{~min}$ and estimated blood loss was less than $50 \mathrm{ml}$. A single dose of $120 \mathrm{mg}$ of Gentimycin was given intraoperatively and ceftriaxone $1 \mathrm{~g}$ daily given for $48 \mathrm{~h}$ intravenously post-operatively. The patient had an uncomplicated post-operative period, had an indwelling catheter left in situ for 5 days and was discharged home 6 days post-operatively. The pathology report confirmed endometriosis throughout the bladder and ureter.

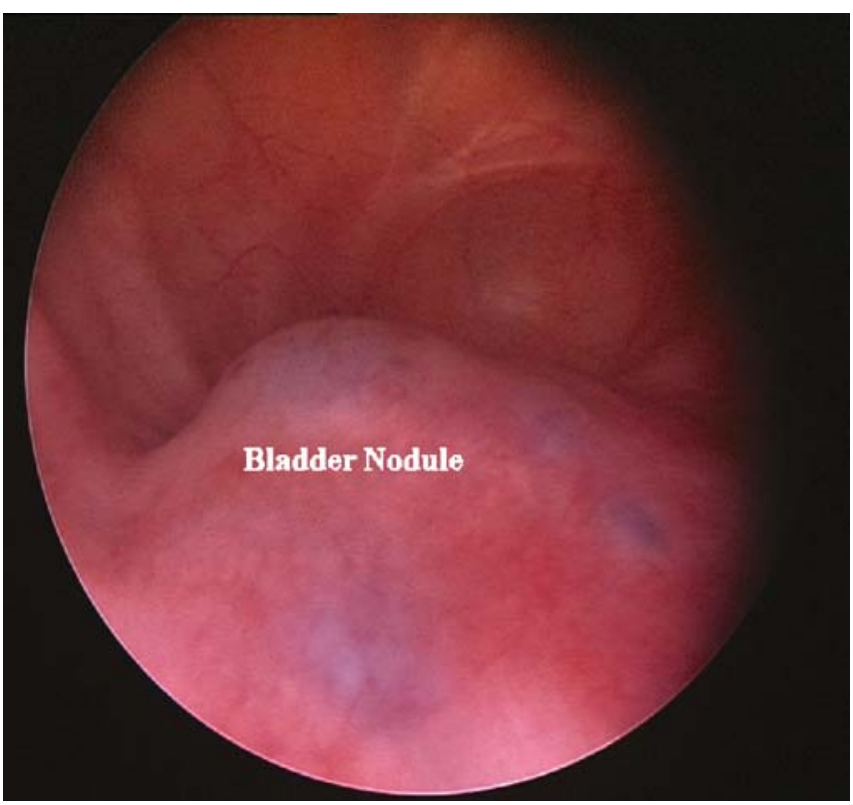

Fig. 2 Dissected ureter

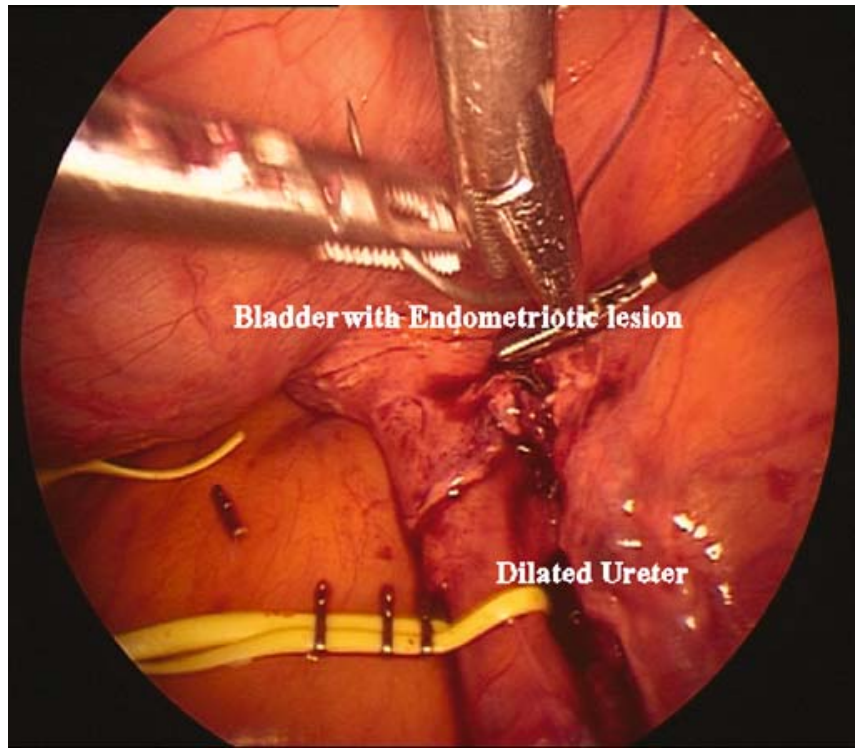

Fig. 3 Endometriotic portion of ureter excised

A post-operative cystogram was done 3 weeks after discharge and showed no filling defects in the bladder and contrast going up the stent. The double-J stent was removed 6 weeks post-operatively and an intravenous urethrogram (IVU) was performed and normal. The patient remained pain free at the 6-month follow-up.

\section{Discussion}

The urinary tract is affected in approximately $2 \%$ of women with endometriosis [1]. When the ureter is involved it appears to affect the extrinsic aspect as opposed to the intrinsic aspect at a ratio of about four to one [7, 8]. Endometriosis is often a progressive, infiltrative disease

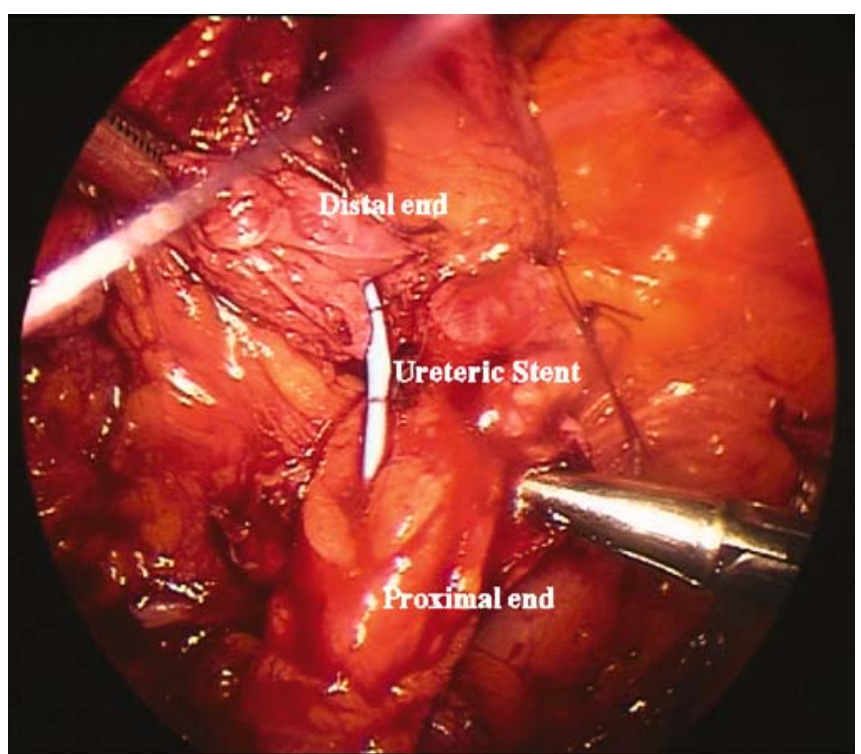

Fig. 4 Psoas hitch, to allow a tension-free anastomosis 


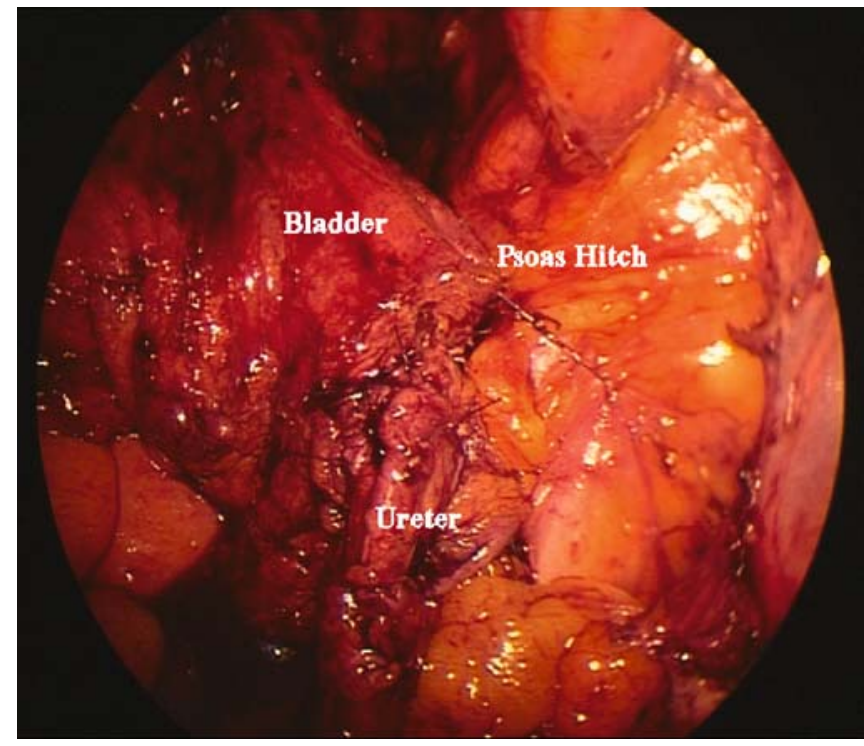

Fig. 5 Suturing of neocystotomy

associated with dense fibrosis of the surrounding tissue. For this reason early diagnosis and treatment may be necessary to avoid loss of renal function if ureteric obstruction ensues. In ureteric endometriosis obstruction may be asymptomatic [2-5] and silent loss of renal function has been reported to be as high as $25-43 \%$ [6]. Radiological modalities such as IVU, CT cystogram and magnetic resonance imaging (MRI) although helpful in demonstrating ureteric obstruction, hydronephrosis, lesion locality and renal damage are non-specific in the diagnosis of renal tract endometriosis. The gold standard remains endoscopy and tissue biopsy.

This procedure has only been performed laparoscopically in very small numbers. This case offers further support to the laparoscopic approach of a procedure traditionally performed by laparotomy. It is important that the vesicoureteric anastomosis is tension free and where this is not the case a psoas hitch or Boari flap may help achieve this [12]. The first case of laparoscopic psoas hitch for infiltrative ureteric endometriosis was described by Nezhat et al. in 1999 [1]. Prior to this the first laparoscopic neoureterocystostomy was performed by Ehrlich in 1993 [9] and the largest series of laparoscopic ureteric reimplantation is in children performed by Lakshmanan and Fung.

Laparoscopy offers a low post-operative morbidity, allows a magnified, clear view of the pelvis and ureter facilitating precise, safe anatomical dissection and excision of pathology in experienced hands. The development of expertise in excision of endometriosis and other advanced laparoscopic techniques [10, 11], particularly where laparoscopic suturing is used such as pelvic floor repair, hysterectomy and Burch procedures, means that the laparoscopic approach to neoureterocystostomy, ureteric implantation and psoas hitch is feasible. However, we would stress that it should only be undertaken by gynaecologists and urologists with advanced laparoscopic training and experience. Where endometriosis is the

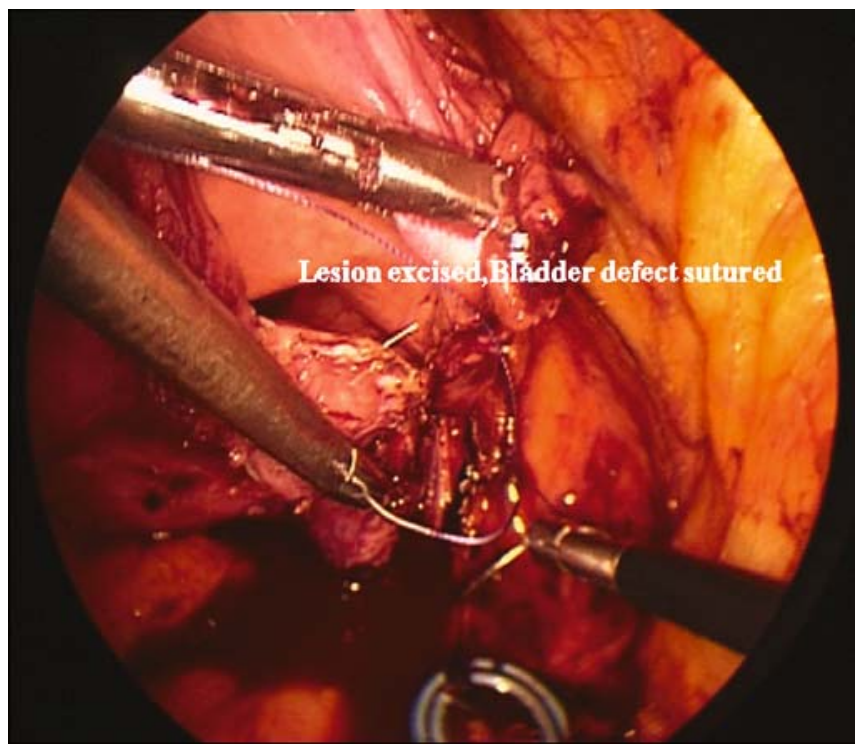

Fig. 6 Reanastomosis of ureter

underlying disease process necessitating the operation ideally the operation should be performed as part of a multidisciplinary approach.

\section{References}

1. Nezhat CH, Nezhat FR, Frieha F, Nezhat CR (1999) Laparoscopic vesicopsoas hitch for infiltrative ureteral endometriosis. Fertil Steril 71:376-379

2. Esen T, Akinci M, Ander H, Tunc M, Tellaloglus S, Narter I (1990) Bilateral ureteric obstruction secondary to endometriosis. Br J Urol 66:948-949

3. Moore JG, Hibbard LT, Growdon WA, Schifrin BS (1979) Urinary tract endometriosis: enigmas in diagnosis and management. Am J Obstet Gynecol 134:162-172

4. Kerr WS (1966) Endometriosis involving the urinary tract. Clin Obstet Gynecol 9:331-357

5. Koszczuk JC, Fogliette M, Perez J, Dono FV, Tomas RJ (1989) Urinary tract endometriosis. J Am Osteopath Assoc 1:83-89

6. Stillwell TJ, Kramer SA, Lee RA (1986) Endometriosis of the ureter. Urology 28:81-85

7. Patel A, Thorpe P, Ramsay JWA, Shepard JH, Kirby RS, Hendry WF (1992) Endometriosis of the ureter. Br J Urol 69:495-498

8. Langemeade CF (1976) Pelvic endometriosis and ureteral obstruction. Am J Obstet Gynecol 122:463-469

9. Ehrlich RM, Gershman A, Fuchs G (1994) Laparoscopic vesicoureteroplasty in children: initial case reports. Urology 43:255-261

10. Canis M, Bruhat MA, Pouly JL, Cooper MJM, Wattiez A, Manhes H (1995) Techniques for ablation and excision of endometriosis. In: Nezhat CR, Berger GS, Nezhat FR, Buttram VC Jr, Nezhat CH (eds) Endometriosis: advanced management and surgical techniques. Springer, Berlin Heidelberg New York, pp 85-94

11. Nezhat C, Nezhat F, Luciano AA, Siegler AM, Metzger DA, Nezhat CH (eds) (1995) Laparoscopic excision of endometriosis. In: Operative gynecologic laparoscopy: principles and techniques. McGraw-Hill, New York, pp 121-147

12. Turney-Warick R, Worth PHL (1969) The psoas hitch procedure for replacement of the lower third of the ureter. Br J Urol $41: 710-714$ 